\title{
Modulation of Slow Cortical Potentials by Instrumentally Learned Blood Pressure Responses
}

\author{
THOMAS ElBERT', LARRY E. ROBERTS ${ }^{2}$, \\ WERnER LUTZENBERGER ${ }^{3}$, AND NIELS BIRBAUMER ${ }^{3,4}$ \\ 'Institut für Experimentelle Audiologie, University of Münster, Germany. \\ 'Department of Psychology. McMaster University, Hamilton, Ontario, Canada, \\ 'Department of Psychology. University of Tübingen. Tübingen, Germany, and \\ 'Università Degli Studi, Dipartimento di Psicologia Generale, Padova, Italy
}

\begin{abstract}
We assessed whether instrumentally-learned pressor responses inhibit electrocortical activity, as predicted by learning theories of idiopathic hypertension. Subjects received beat-by-beat feedback for increases and decreases in mean arterial pressure measured from the finger (Peñáz method). Slow potentials were recorded from the midsagittal line during the final training session. Also recorded at this time were heart rate, eye movements, respiration, and post-session verbal reports of the subject's control strategies. Thirteen of 14 subjects differentiated blood pressure increases and decreases at $p<.05$ or better during the final session (within-subject discriminative operant procedure). Slow potentials were less negative on blood pressure increase compared to decrease trials at all midsagittal sites $(p<.02)$, indicating relative cortical inhibition by pressor responses. This effect occurred even though subjects reported tensing of muscles on increase trials $(p<.01)$, a behavioral activity previously associated with augmented rather than diminished cortical negativity. On increase trials slow potentials shifted toward positivity just prior to heart rate deceleration (the latter effect confirming activation of the baroreceptors).
\end{abstract}

DESCRIPTORS: Blood pressure biofeedback, Slow cortical potentials, Hypertension, Fin.A.Pres., Verbal reports, Instrumental learning.

As part of their role in cardiovascular regulation, the baroreceptors appear to exert a generalized inhibitory effect on the brain. Inhibition of electrocortical responses has been documented following stimulation of the baroreceptors by several methods, including (1) surgically induced renal hypertension (Garsik, Low, \& Whitehorn, 1983), (2) infusion of vasoconstrictive drugs (Larbig, Elbert, Rockstroh, Lutzenberger, \& Birbaumer, 1985), and (3) stretching of the carotid sinus by suction applied to the neck (Elbert et al., 1988). Activation of the baroreceptors by one or more of these methods has also been reported to induce sleep (Dworkin, 1988;

This experiment was funded by the Deutsche Forschungsgemeinschaft (EL101/3, SFB 307) and the NATO Division of Scientific Affairs (0530/87). We thank Johannes Kräemer for data collection, and Brigitte Rockstroh and Harald Rau for their helpful comments.

Address request for reprints to: Thomas Elbert, Institut für Experimentelle Audiologie, Kardinal-von-Galen-Ring 10, Mûnster D4400, Germany, or Larry E. Roberts, Department of Psychology, McMaster University, Hamilton, Ontario, Canada L8S 4K1.
Koch, 1937) and to attenuate behavioral reactions to painful and aversive stimuli (Dworkin, Filewich, Miller, Craigmyle, \& Pickering, 1979; Randich, 1982). Antinociceptive effects appear to require an intact baroreceptor innervation (Dworkin et al., 1979; Randich \& Hartunian, 1983) and have been reported in human subjects when arterial pressure is in the borderline hypertensive range (Elbert et al., 1988; Larbig et al., 1985).

Evidence for cortical and behavioral inhibition arising from the baroreceptors led Dworkin et al. (1979) to propose that some cases of idiopathic hypertension might be attributable to instrumental learning. According to this hypothesis, hypertension results when, in the presence of noxious stimulation, blood pressure increases are reinforced over a sufficient period of time by the cortical-inhibitory effects of baroreceptor firing. This analysis is consistent with evidence showing that increases in blood pressure can be learned instrumentally through biofeedback (Harris, Gilliam, Findley, \& Brady, 1973; Plumlee, 1968; Victor, Weipert, \& Shapiro, 1984). However, it is not known whether pressor responses induced by this method are suf- 
ficient to produce the hypothesized cortical inhibitory effect.

In this study we examined the electrocortical effects of instrumentally learned blood pressure responses. To assess cortical activation, we recorded slow (dc) potentials from the scalp while subjects increased and decreased their blood pressure under conditions of biofeedback. Previous research has shown that negative-going changes in slow potentials signify cortical activation and accompany improved performance on a wide range of perceptual and sensorimotor tasks (see Birbaumer, Elbert, Canavan, \& Rockstroh, 1990, for a review). Therefore, in accordance with the baroreceptor theory of learned hypertension, a suppression of negativity (i.e., a positive-going shift in slow potentials) was predicted when the baroreceptors were activated by pressor responses acquired through biofeedback. This result would also corroborate previous studies, which have found that mechanical stimulation of the baroreceptors reduces slow cortical negativity (Elbert et al., 1988; Rau et al., 1988).

A further goal of the study was to determine how subjects achieve control of their blood pressure during biofeedback. As described by Dworkin (1984, 1988), the baroreceptor hypothesis suggests that highly specific blood pressure responses are acquired through instrumental learning, and that the learning of such responses occurs outside of conscious attention. However, little is presently known about the response patterns that occur during biofeedback for blood pressure, or whether these response patterns are explicitly attended to during learning. We addressed these questions by gathering verbal reports of the subject's control strategies after biofeedback training. To ensure the validity of the verbal report data, we used a biofeedback procedure that allowed us to separate self-report arising from the subject's memory of behavior related to feedback occurrence, from self-report arising from other factors that can influence retrospective report in a feedback situation (e.g., reporting biases induced by task instructions, features of the training environment, or orientation of the feedback display). Analysis of the verbal report for knowledge of feedback behavior also provided a test of whether that behavior had been processed in attention.

\section{Method}

\section{Subjects}

Sixteen healthy normotensive students $(9$ men and 7 women, aged 21-31 years, all nonsmokers) completed feedback training. Two male subjects were subsequently excluded from the analysis due to artifact in the EEG in more than one-third of the trials. Subjects earned 2 deutschmarks for each correct response and lost the same amount for each incorrect response, but were paid not less than 30 nor more than 100 deutschmarks per session (mean payment 44 deutschmarks, equivalent to approximately $\$ 28$ U.S. at the time of the study).

\section{Physiological Recordings}

Arterial blood pressure was monitored continuously by means of an inflated cuff (Ohmeda Fin.A.Pres.) attached around the second phalanx of the left middle finger. Changes in blood volume consequent on each heartbeat were sensed by a photoplethysmyograph and fed to a purpose-built servo-mechanism that regulated cuff pressure to equal that of the finger's arteries. This method of recording blood pressure was developed and validated by Wessling, Settels, and de Wit (1986) following an earlier suggestion by Peñáz (1973). Because transmural pressure was maintained near zero by servo-regulation, the Fin.A.Pres. transducer could be worn comfortably, although pulsatile sensations produced by the beating heart were noticeable and faded in and out as pressure in the cuff was adjusted. The output of the Ohmeda device was sampled at $100 \mathrm{~Hz}$ by a PDP $11 / 73$ computer. A sample blood pressure recording obtained on one increase and one decrease feedback trial is shown in Figure 1, to illustrate the temporal resolution of this measurement technique.

The EEG was recorded along the midsagittal line $\left(\mathrm{F}_{\mathrm{PZ}}, \mathrm{F}_{\mathrm{Z}}, \mathrm{C}_{\mathrm{Z}}, \mathrm{P}_{\mathrm{Z}}\right.$, and $\left.\mathrm{O}_{\mathrm{Z}}\right)$ according to the international $10-20$ system. Readings were referred to the midpoint of a $10 \mathrm{Kohm}$ shunt attached between the earlobes. The skin at each site was cleaned with alcohol and abraded with a sterile lancet to reduce impedance below $5 \mathrm{Kohms}$. Grass $\mathrm{Ag} / \mathrm{AgCl}$ disk electrodes (chlorided before each session and coated with EC2 paste) were then affixed. A single ground electrode was attached to the neck. The EEG was amplified by a Nihon-Kohden amplifier specially manufactured to have a time constant of $10 \mathrm{~s}$. Amplifier output was sampled at $100 \mathrm{~Hz}$ and filtered by the method of Elbert and Rockstroh (1980) to give the DC component. We will refer to the filtered EEG channels as slow potentials.

Additional measures were as follows (see Elbert et al., 1988 for further details). The electrocardiogram
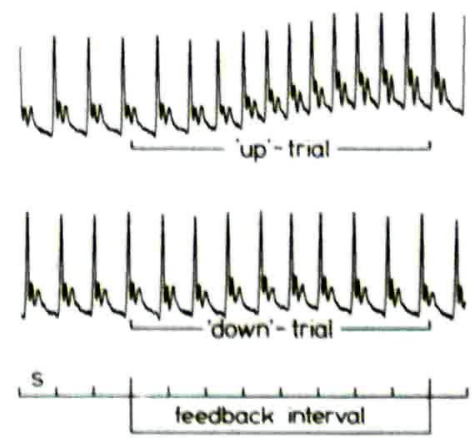

Figure 1. Sample blood pressure recording by the Fin.A.Pres. method on one increase and one decrease feedback trial. 
(ECG) was recorded from the lower rib cage (leads $V_{1}$ and $V_{5}$ ) and sampled at $100 \mathrm{~Hz}$ by the computer. Heart rate was calculated for successive .1-s intervals by weighting each beat according to the proportion of the interval it occupied. Respiration was measured from a pneumatic belt (Beckman model $872-428$ ) placed around the lower chest. Vertical and radial electrooculograms (EOGs) were derived from two electrodes attached $0.5 \mathrm{~cm}$ below each eye. The average potential of these two electrodes was subtracted from the $F_{\mathrm{PZ}}$ electrode to obtain the vertical EOG, and from the average earlobe potential to obtain the radial EOG (after Elbert, Lutzenberger, Rockstroh, \& Birbaumer, 1985). These measures were used to screen the EEG channels for eye movement artifacts. Blood pressure was also obtained from the left arm before and after each session, using an automated Riva-Rocci cuff.'

\section{Training and Test Sessions}

There were two types of sessions, training and test. Training sessions were given daily until the subject met a prespecified criterion for learning (stated below), or until four sessions had been completed. A single test session was administered the following day. Subjects therefore received a minimum of two and a maximum of five sessions overall, depending on their rate of learning. Sessions lasted about 1 hour and were conducted in a sound-attenuated, electrically shielded room.

Training and test sessions differed only with regard to the response measures that were taken. Heart rate and blood pressure from the finger were recorded continuously during training sessions. Electrodes were also affixed at $C_{Z}$ and the earlobes during these sessions, but no measures were taken from these sites. For the test session, recordings of respiration and slow cortical potentials at all midsagittal sites were added. Verbal reports were also collected after the end of the test session (see below).

On the first training session subjects received written instructions informing them that two responses would be trained. Subjects were not told what the responses were (increases and decreases in blood pressure) or how to control them, although they were in-

'Baseline systolic blood pressure was found to be lower when measured by the Riva-Rocci cuff $(131.6 \mathrm{mmHg})$ than when measured by the Fin.A.Pres. method (142.3 $\mathrm{mmHg}$ ), $t(15)=4.3, p<.01$ (average of all measures in the first two training sessions). The reverse relation was found for diastolic blood pressure, 82.5 versus $77.1 \mathrm{mmHg}$, respectively, $t(15)=2.9, p<.01$. These differences appear to be explicable, at least in part, in terms of the lower position of the finger compared to the arm, and to the tendency of the Rica-Rocci cuff method to underestimate systolic blood pressure. The product-moment correlation between the two techniques was .80 for systolic and .70 for diastolic baselines, respectively $(p<.01)$. Baseline blood pressures are not discussed further in this article. formed that the two responses were "opposites."2 Subjects were asked not to tense their muscles or move their eyes or tongue. No information was given about the purpose of any physiological transducer. Eight practice trials with feedback were given at the outset of the first session, to familiarize subjects with the task. However, no data were retained from these trials.

\section{Procedure}

Continuous visual feedback was provided for beatby-beat changes in mean arterial blood pressure on trials of 8-s duration. The feedback stimulus was the outline of a small rocket ship that appeared on a 30 $\times 40 \mathrm{~cm}$ TV screen situated $2 \mathrm{~m}$ in front of the subject at eye level. The rocket moved back and forth in a horizontal plane through a gap formed by two vertical bars, one bar above the gap and one bar below it. The bars were situated slightly to the left of center on the TV screen. Trials were designated by presentation of the rocket in the gap, together with the letter $A$ or $B$ at the right boundary of the screen. The subject's task was to move the rocket out of the gap toward the letter on each trial. Assignment of the blood pressure responses (increase and decrease) to the discriminative stimuli (the letters $A$ and $B$ ) varied randomly among subjects. It should be noted that correct blood pressure changes moved the rocket toward the right (success feedback) and incorrect changes moved it to the left (error feedback) on A as well as B trials. Subjects therefore attempted the same feedback excursions on $A$ and $B$ trials, but the blood pressure responses (increase/ decrease) that produced these excursions were different between the A and B trial types. To preserve consistency with previous research in which this feedback procedure was applied to slow cortical potentials rather than to blood pressure (Roberts, Birbaumer, Rockstroh, Lutzenberger, \& Elbert, 1989), the position of the rocket remained fixed at the starting point for the first second of each feedback trial.

Systolic and diastolic arterial pressures (maximum and minimum values, respectively, of the Fin.A.Pres. recording) were determined on-line for each $R-R$ interval. Movements of the rocket on each feedback trial were a linear function of the integral of mean blood pressure $(2 / 3$ diastolic plus $1 / 3$ systolic), referred to a 4$s$ pretrial baseline and updated with each heartbeat. If the mean change in pressure exceeded $5 \mathrm{mmHg}$, the outline of the rocket became filled, signalling that the subject had earned or lost a "win" point worth 2 deutschmarks (depending on whether the rocket was on the success or failure side of the starting gap). When

In earlier studies using isomorphic feedback methods (reviewed in Roberts, 1989, and including Roberts, Birbaumer, Rockstroh, Lutzenberger, \& Elbert, 1989), subjects were not informed that the two feedback responses were opposites. This procedural variation does not affect the logic of the verbal report analysis (see Roberts. Williams, Marlin, Farrell, \& Imiolo, 1984). 
subjects achieved a net score of 15 win points (algebraic total on increase trials plus algebraic total on decrease trials) within a training session, the test session was scheduled.

To assess control of responding in the absence of feedback, "transfer" trials were included on which the letter $A$ or $B$ was presented but feedback was removed. Subjects were told that they should produce the required $A$ or $B$ response as best they could on transfer trials, even though feedback and win points would not be displayed to tell them how well they had done. Each session began with a block of 30 feedback trials (15 A and $15 \mathrm{~B}$ ) followed by a block of 20 transfer trials ( 10 of each kind). This sequence was then repeated once to give a total of 100 trials for the session. A brief rest was scheduled between each of the four blocks (two feedback and two transfer). Within blocks, A and B trials were given in a mixed order. The time lapse from the end of one trial to the beginning of the next pretrial baseline varied randomly between 4 and $12 \mathrm{~s}$.

At the end of the test (final) session, subjects were asked without prior notification to complete two questionnaires. The first questionnaire asked subjects to describe in writing the strategies they used to achieve success on A and B trials (success behavior and error behavior separately; open-ended replies). A second questionnaire was then given which required that subjects rate on 7-point scales $(3=$ a great deal, $-3=$ the opposite pole; midpoint of $0=$ no change) the degree to which the following specific activities were used to achieve success on A and B trials: 1) tense muscles versus relax muscles; 2 ) prepare a rapid novement versus remaining still; 3) fast, deep breathing versus slow, shallow breathing; 4) suspended versus normal breathing: 5) emotional tension versus relaxation; 6) imagining aversive versus pleasant situations; 7) concentration versus distraction; 8) observing versus not observing the rocket; 9) thinking versus not thinking about a strategy; and 10 ) imagining versus not imagining concrete objects. An additional scale required that subjects rate their perceived success on $A$ and $B$ trials $(7=$ very successful; $1=$ not successful at all).

\section{Analysis of Physiological Data}

Continuous physiological measures (systolic, diastolic, and mean blood pressure; heart rate; respiration; EEG and EOG) were collapsed to $0.1-\mathrm{s}$ intervals by a phase-free digital filter. Change scores were then computed for each measure by subtracting the mean for the last $7 \mathrm{~s}$ of the trial from the mean observed during the last second of the pretrial period (this pretrial referent was adopted by previous studies for analysis of slow-potential shifts; see Roberts et al., 1989). Trials containing (1) excessive vertical or radial eye movements (EOG $>70 \mu \mathrm{V})$, (2) extraneous EEG shifts $(>70$ $\mu \mathrm{V})$ in any channel, or (3) other obvious artifacts were rejected from the analysis. Twenty percent of the total of 1400 trials was rejected for one or more of these reasons.
Change scores for each physiological response were subsequently averaged over blood-pressure increase and decrease trials separately, in each feedback and transfer block. This yielded 8 summary measures per subject in each session (increase and decrease trials $X$ 2 feedback blocks $\times 2$ transfer blocks) for each physiological variable. Unless otherwise indicated, these data provided the basis for statistical tests (MANOVAs and Student's $t$ ). It was often convenient to summarize the effect of feedback training on responding by subtracting $A$ and $B$ trials from one another (increase minus decrease). Measures computed this way are referred to as "bidirectional."

\section{Analysis of Verbal Reports}

The open-ended verbal reports (Questionnaire \#1) were analyzed following the method of Roberts et al. (1989). Ten judges were recruited from students and staff of the Institute of Psychology, University of Tübingen. These judges were given each subject's openended report of success and failure behavior on $\mathrm{A}$ and B trials. On the basis of these reports, judges were asked to decide whether the subject had been trained to increase blood pressure on A trials and decrease it on B trials, or the reverse. To assist their decisions (which were made independently), we informed judges of earlier evidence relating cardiovascular responses to somatomotor and respiratory action under nonfeedback conditions (Abboud \& Thames, 1983; Sharpey-Schafer, 1963). However, judges had no knowledge of the subject's measured performance or of the assignment of the blood pressure responses to $\mathrm{A}$ and B trials. If judges discerned an alternative basis on which to assign the verbal reports, they were invited to use it.

The ratings that subjects gave on the scales assessing specific response strategies (Questionnaire \#2) were analyzed by computing bidirectional differences for each subject on each scale. It was then determined whether the bidirectional scores for each scale differed significantly from zero (Wilcoxon's tests).

In the feedback procedure of this study, A and B trials differed only with regard to the behavioral activities or mental states for which feedback was provided. Significant bidirectional differences on a verbal report scale therefore indicated that the activity assessed by that scale (or behavior entailed by that activity) was part of the neuropsychological process differentiated by feedback, and that subjects were aware of this activity or at least one of its constituents after feedback training. Correct assignment of the open-ended verbal reports by the judges similarly implied an awareness of behavior associated with feedback, provided that tanget difficulty was not the basis of the judges' decisions. It should be noted that these statements apply only to analyses of group data (e.g., of central tendency or correlation) in which the presence of multiple independent subjects diminished the likelihood that a verbal report was adventitiously associated with a particular trial type. Verbal reports of 
individual subjects are open only to descriptive interpretation, because the content of the report could have been attributable to guessing (see Roberts et al., 1984).

\section{Results}

The plan for statistical analysis was adapted from previous research in which a similar feedback method was applied to different responses (Roberts et al., 1989). Unless otherwise stated, statistical tests were two-tailed ( $\alpha \leq .05$; one-tailed tests accepted only when directional predictions were made).

\section{Acquisition of Blood Pressure Control}

Of the 14 subjects included in the experiment, 8 met the criterion for learning ( 15 net win points) in the first training session. Only 1 subject failed to achieve criterion within 4 training sessions. Figure 2 shows how net win points (top panel) and bidirectional differences in mean blood pressure (bottom panel) changed over sessions, when subjects were divided into subgroups according to the total number of sessions they received $(8,3,1$, and 2
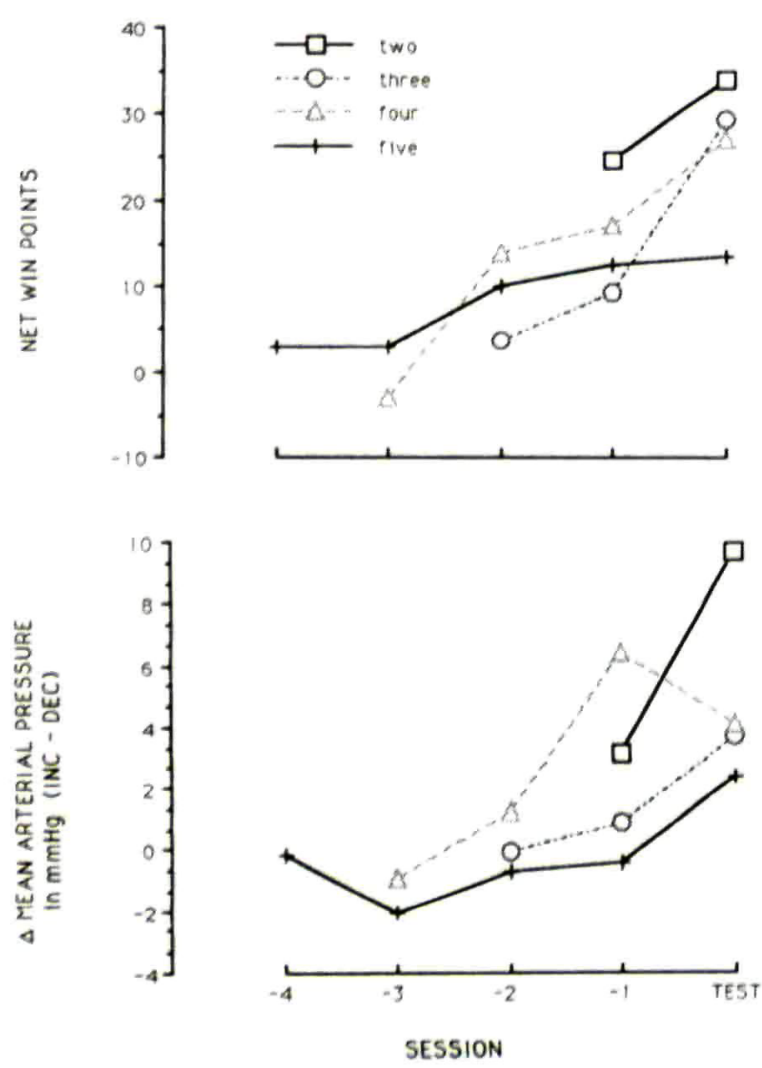

Figure 2. Changes in net win points (top) and bidirectional differences in mean blood pressure (bottom) over sessions. Subjects were divided into independent subgroups according to the total number of sessions they received. Blood pressure is reported for the first feedback block in each session, to illustrate initial performance. subjects received $2,3,4$, and 5 sessions, respectively, including the test session). Analysis of the data collapsed over subgroups showed that bidirectional differences in mean blood pressure (the feedback variable) were larger in the concluding feedback block of the test session than in the first feedback block of the experiment, $t(13)=3.60, p<$ .01. Similar results were obtained for mean blood pressure contrasted between the corresponding transfer blocks, $t(13)=2.89, p<.02$, and for net win points contrasted between the test and initial training sessions, $t(13)=3.78, p<.01$. Of the 14 subjects studied, 13 differentiated significantly between blood pressure increases and decreases during the test session (within-subject $t$-tests, feedback and transfer combined, one-tailed). It should be noted that differentiation of the two responses implies acquisition, because in the current procedure the blood pressure responses were identified by feedback alone.

These findings establish that subjects were successful at learning to control blood pressure. Results presented subsequently are derived from the test session, during which the subject's performance was examined in greater detail.

\section{Cardiovascular Responses}

Changes in systolic and diastolic blood pressure on increase and decrease trials are shown in the upper rows of Figure 3, for each of the four blocks comprising the test session (two feedback and two transfer). Inspection of these data shows that subjects were successful at increasing blood pressure on trials requiring this performance, in each trial block. Systolic pressure peaked at about +10 $\mathrm{mmHg}$ (diastolic $+8 \mathrm{mmHg}$ ) approximately $5 \mathrm{~s}$ into the trial, and declined thereafter. However, subjects were less successful at decreasing blood pressure on trials requiring this response. Small increases in blood pressure were observed on decrease trials early in the session, but these increases shifted in the reinforced direction as training progressed.

These findings were corroborated by statistical analyses of the systolic, diastolic, and mean blood pressure changes (the latter not shown in Figure 3). MANOVAs revealed an effect of direction (increase/decrease) on systolic blood pressure, $F(1,13)=9.5, \quad p<.01$, diastolic blood pressure, $F(1,13)=18.4, p<.01$, and mean blood pressure, $F(1,13)=16.1, p<.01$. A Direction $\times$ Blocks (first/ second) interaction was also found for diastolic blood pressure, $F(1,13)=8.5, p<.05$, and for mean blood pressure, $F(1,13)=5.2, p<.05$. These interactions appeared to be due in part to an improvement on decrease trials across blocks. Simple $t$-tests corroborated an improvement in decrease perform- 


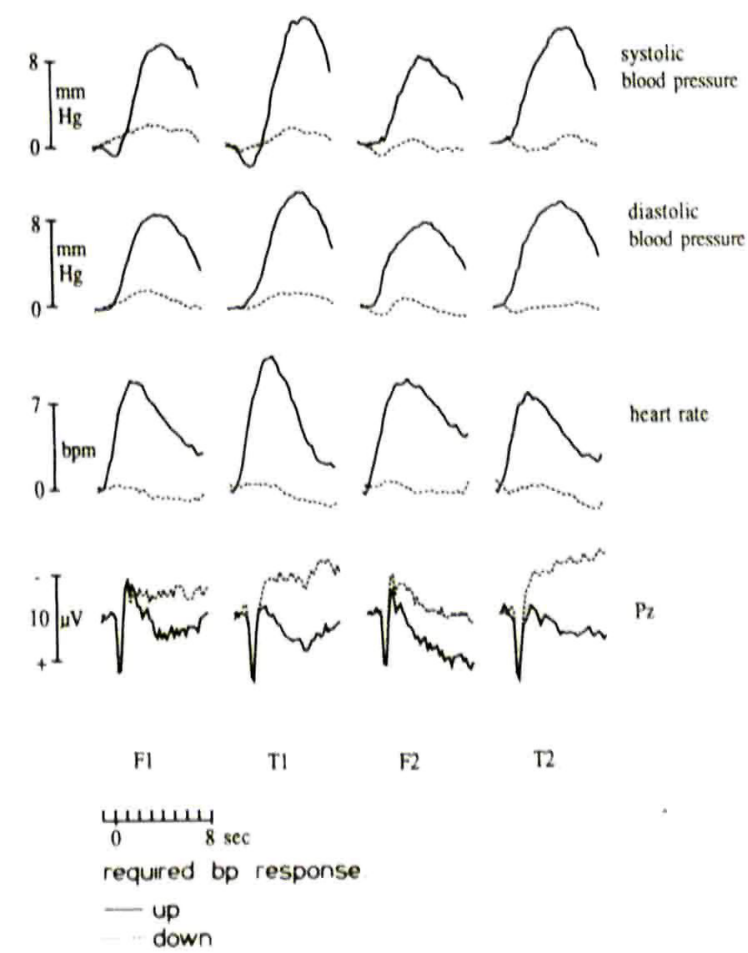

Figure 3. Cardiovascular responses (top three traces) on increase and decrease trials in successive feedback $(F)$ and transfer $(T)$ blocks. Slow potentials at $P_{Z}$ are shown in the lower trace. Solid lines are blood-pressure increase trials, broken lines blood-pressure decrease trials.

ance across feedback blocks for diastolic blood pressure, $t(13)=3.37, p<.01$ ), and for mean blood pressure, $t(13)=3.03, p<.01$. Also, diastolic and mean blood pressure decreased from the pretrial baseline on decrease trials of the second feedback block, $t(13)=-2.02$ and -2.11 , respectively $(p<.05$, one-tailed tests), whereas increases in blood pressure occurred during the initial feedback series. Similar results were obtained on the transfer blocks. Neither the main effect of feedback/transfer, nor any interaction involving this variable, was significant for any blood pressure measure.

Changes in heart rate are shown in the third row of Figure 3. A pronounced tachycardia commenced at the onset of increase trials, in all feedback and transfer blocks. This response peaked at about +8 bpm approximately $2 \mathrm{~s}$ into the trial, after which a deceleration occurred. On decrease trials a small bradycardia was visible, but this response was not significant for the session as a whole. MANOVA revealed only an effect of direction, $F(1,13)=21.8$, $p<.01$.

\section{Slow Potentials}

Changes in slow potentials produced by increasing and decreasing blood pressure are shown in Fig-

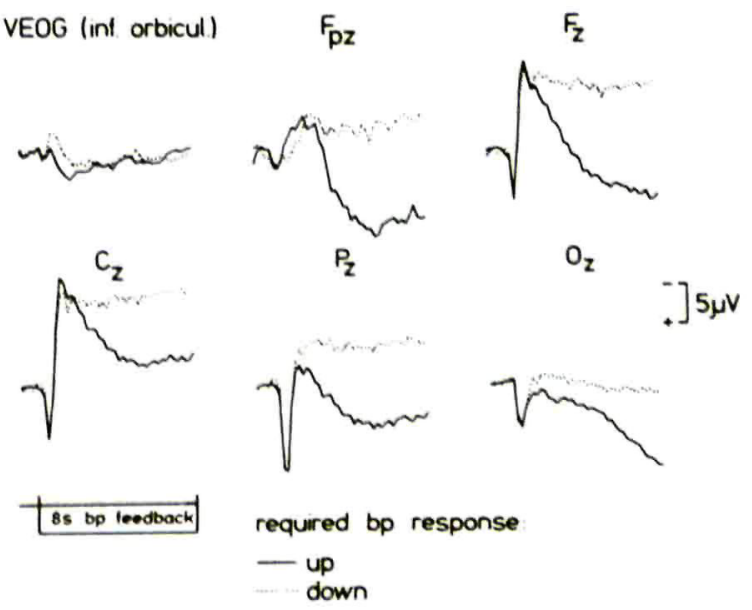

Figure 4. Changes in slow potentials and eye movements (VEOG) on increase and decrease trials. Solid lines are blood-pressure increase trials, broken lines blood-pressure decrease trials.

ure 4. EOGs consequent on vertical eye movements are also depicted (VEOG, a possible source of spurious slow-potential shifts at midline locations). These data are averaged over all feedback and transfer trials of the test session.

Inspection of Figure 4 shows that VEOGs did not differ between the two directions of training. However, slow potentials differed bidirectionally at each midsagittal site. The direction of this difference was toward positivity on trials in which pressor responses were produced. MANOVA of the slow-potential data confirmed a main effect of direction, $F(1,13)=6.7, p<.02$, and also of sites, $F(4,52)=5.6, p<.01$. The latter effect was due to larger negative shifts occurring at the frontal $\left(\mathrm{F}_{2}\right)$ and central $\left(\mathrm{C}_{\mathrm{Z}}\right)$ locations than at $\mathrm{F}_{\mathrm{PZ}}, \mathrm{P}_{\mathrm{Z}}$, and $\mathrm{O}_{\mathrm{Z}}$ (the well-known frontal and central dominance). The time course of the slow-potential differentiation was similar at the various midline sites, although the direction of the differentiation appeared to have been initially reversed at $F_{\mathrm{Pz}}$. Interactions attributable to site, and effects involving feedback/ transfer and blocks, were not significant in the slowpotential analysis.

Between-subject correlations were computed between slow-potential and blood pressure responses (bidirectional differences) on feedback trials. These correlations, all of which were positive, declined monotonically with increasing proximity to $\mathrm{C}_{\mathrm{Z}}$. However, only the largest correlation, $r=.56$ at $\mathrm{O}_{\mathrm{z}}$, reached significance $(p<.05)$.

\section{Verbal Reports}

At the end of the test session, subjects described their performance on response scales assessing par- 
Table 1

Ratings on the verbal report scales

\begin{tabular}{|c|c|c|c|}
\hline \multirow[b]{2}{*}{ Scale } & \multicolumn{3}{|c|}{ Scale Rating } \\
\hline & Increase & Decrease & Difference \\
\hline $\begin{array}{l}\text { 1. Muscle tension } \\
\text { 2. Prepare rapid }\end{array}$ & $1.64^{* *}$ & $-1.57^{* *}$ & $3.21^{* *}$ \\
\hline movement & $1.69^{*}$ & $-1.57^{* *}$ & $3.26^{* *}$ \\
\hline $\begin{array}{l}\text { 3. Deep fast breath } \\
\text { 4. Stopped }\end{array}$ & $0.93^{* *}$ & $-1.50^{* *}$ & $2.43^{*}$ \\
\hline $\begin{array}{l}\text { breathing } \\
\text { 5. Emotionally }\end{array}$ & 0.07 & -0.64 & 0.91 \\
\hline $\begin{array}{c}\text { tense } \\
\text { 6. Aversive }\end{array}$ & $1.21^{*}$ & -0.64 & $1.85^{*}$ \\
\hline emotion & 0.14 & $-1.71^{\bullet *}$ & 1.85 \\
\hline $\begin{array}{l}\text { 7. Concentration } \\
\text { 8. Think about }\end{array}$ & $2.21 * 0$ & $1.21^{\star *}$ & 1.00 \\
\hline $\begin{array}{c}\text { rocket } \\
\text { 9. Think about }\end{array}$ & $1.86^{*}$ & 0.71 & 1.15 \\
\hline strategy & 0.77 & 0.69 & 0.08 \\
\hline 10. Imagery & 1.36 & 0.43 & 0.93 \\
\hline
\end{tabular}

Note. Subjets rated the degree to which each of the above response activities was used to achieve success on increase and decrease $(\mathrm{A} / \mathrm{B})$ trials, using a seven-point scale $(3=\mathrm{a}$ great deal, -3 - the opposite pole; midpoint of 0 - no change.)

$* p<.05, * p<.01$ (Wilcoxon Tests).

ticular control strategies on increase and decrease trials. Inspection of the results given in Table 1 shows that several scales differentiated significantly between the two trial types. Muscular activity (Scales 1 and 2), augmented breathing (Scale 3), and emotional arousal (Scale 5) were reported on trials on which an increase in blood pressure was required, and responses of the opposite type when decreases were called for. Increased concentration and attention to the rocket were also indicated (Scales 7 and 8 ), but these activities were reported during blood pressure increases as well as decreases and did not differ between the two directions. Between-subject correlations were computed between bidirectional differences on the scales and bidirectional differences in blood pressure. The largest correlation was obtained for the scale assessing deep, fast breathing $(\rho=.29)$, but none of the correlations were significant.

Prior to completing the response scales, subjects described their response strategies in an open-ended verbal report. The validity of these reports was assessed by determining whether judges were able to assign the blood pressure targets (increase/decrease) to the trial cues $(\mathrm{A} / \mathrm{B})$ at a frequency exceeding chance, on the basis of the success and error behaviors mentioned by the subjects. Judges assigned an average of 12 of the 14 subjects correctly, compared to a chance expectation of 7 subjects (Kolmogoroff-Smirnov $D_{\max }=.86, p<.01$ ). Because valid response information was therefore present, the success behaviors that subjects mentioned were tab-
Table 2

Response activities described in the open-ended verbal reports

\begin{tabular}{lll}
\hline & \multicolumn{1}{c}{$\begin{array}{c}\text { Number of Subjects } \\
\text { (total = 14) }\end{array}$} \\
\cline { 2 - 3 } Activity Mentioned & Increase & Decrease \\
\hline $\begin{array}{l}\text { Muscle Tension } \\
\text { Tense muscles; contract } \\
\text { leg muscles toe to hips; } \\
\text { clench teeth }\end{array}$ & & 1 \\
$\begin{array}{l}\text { Somatomotor Relaxation } \\
\text { relax muscles; imagine } \\
\text { fatigue; everything loose }\end{array}$ & 7 & \\
$\begin{array}{l}\text { Respiratory Activation } \\
\text { Hard inhalation; rapid } \\
\text { breathing: stess/release } \\
\text { diaphragm }\end{array}$ & 1 & \\
$\begin{array}{l}\text { Decreased or Regular } \\
\text { Respiration } \\
\text { Slow, shallow breathing: } \\
\text { stopped breathing: regular } \\
\text { breathing }\end{array}$ & & \\
$\begin{array}{l}\text { Arousing Thoughts } \\
\text { Erotic fantasies; stressful } \\
\text { thoughts; imagine } \\
\text { annoying person }\end{array}$ & 4 & \\
$\begin{array}{l}\text { Relaxing Thoughts } \\
\text { Think of seashore, } \\
\text { poetry: imagine white } \\
\text { space }\end{array}$ & & \\
\hline
\end{tabular}

Note. Each category is illustrated by examples from the subjects' verbal reports. Numerical entries are the number of subjects out of 14 reporting strategies in each category, separately for increase and decrease trials. The judges' assignments were used to assess these data statistically (sce text). because the verbal reports for increase and decrease trials were not independent.

ulated for increase and decrease trials separately, and are reported in Table 2 . Tensing of muscles was described by 7 subjects on increase trials (e.g., progressive contraction "of leg muscles from the big toe to the hips"), and respiratory manipulations by 4 subjects (e.g., "hard inhaling and exhaling," and "stress/release diaphragm"). On the other hand, somato-respiratory quiescence was typically reported on decrease trials. Cognitive strategies were also reported by a majority of subjects (e.g., "erotic fantasies" and "pleasant thoughts"), with these differing between increase and decrease trials when classified as either arousing or relaxing. The success of the judges in assigning the open-ended verbal reports did not appear to depend on differences in the difficulty of blood pressure increases and decreases. Although all subjects rated decreases as the more difficult response $(p<.01$, sign test), no subject mentioned differences in target difficulty in the open-ended reports that were seen by the judges.

\section{Respiration}

Respiration was analyzed to determine whether verbal reports of respiratory manipulation were 
corroborated by response measurement. Principal Components Analysis extracted six components when applied to the data (Subjects $\times$ Direction $\times$ Successive .1-s Intervals), but in no case did linear weights determined for these components differ significantly between increase and decrease trials. However, power spectral analysis revealed more total respiratory power on increase compared to decrease trials, $F(1,13)=12.6, p<.01$. Bidirectional differences in total power tended to be higher for subjects reporting respiratory strategies, but this difference did not reach significance.

\section{Discussion}

One purpose of the current study was to determine whether pressor responses learned through biofeedback are sufficient to modulate electrocortical activity through a baroreceptor mechanism. A further goal was to examine the process by which pressor responses are acquired through experience with feedback. We discuss (1) the electrocortical effects of learned pressor responses, and (2) the implications of our findings for theories of the role of learning in idiopathic hypertension.

\section{Electrocortical Effects of Learned Pressor Responses}

Previous research has shown that stimulation of the baroreceptors by extrinsic methods (neck suction, drug infusion, surgically-induced renal hypertension, carotid massage) inhibits electrocortical and behavioral functions. Among the effects documented by these methods are inhibition of learned escape and avoidance behavior (Dworkin et al., 1979), pain responses (Randich \& Hartunian, 1983; Larbig et al., 1985), electrocortical processes including event-related potentials (Garsik et al., 1983), EEG frequency changes (Bonvallet, Dell, \& Hiebel, 1953), and slow cortical potentials (Elbert et al., 1988). In the present study we found that activation of the baroreceptors by an instrumentally learned pressor response also inhibited cortical activity, as measured by a positive-going shift in slow cortical potentials. It is noteworthy that cortical inhibition was observed when the baroreceptors were stimulated intrinsically by a learned response, rather than extrinsically by a surgical, pharmacological, or mechanical intervention.

The temporal relationship that we observed between cardiovascular and electrocortical changes supports the conclusion that differentiation of slow potentials was secondary to activation of the baroreceptors. This relationship can be seen in Figure 3 , where for purposes of illustration slow-potential shifts occurring at $\mathrm{P}_{\mathrm{Z}}$ are contrasted with the cardiovascular changes that occurred in each trial block. Because changes in blood pressure were small and often nonexistent on decrease trials, such trials can be taken to show how electrocortical activity was affected when feedback was processed in the absence of pressure variations. The addition of pressor responses to feedback processing on increase trials would be expected to stimulate the baroreceptors, thus inducing the hypothesized cortical inhibitory effect. In this respect it should be noted that differentiation of slow potentials between increase and decrease trials developed prior to the onset of cardiac deceleration in each trial block. These events and their temporal relation (separated by approximately one heartbeat) are consistent with a baroreceptor-induced modulation of the cardiac pacemaker. Figure 3 also shows that the peak of the pressor response lagged the onset of heart rate deceleration, and was followed by a decrease in pressure as the end of the trial approached. These features of the pressor response are consistent with a slower acting, baroreceptor-induced vasodilation (Mancia \& Mark, 1983). Changes in systolic pressure followed the changes in diastolic pressure by about $1 \mathrm{~s}$ in Figure 3, because a heartbeat was required for determination of the former. ${ }^{3}$

Evidence that positive-going slow potentials signify cortical inhibition (or, more precisely, a withdrawal of cortical excitation, or disfacilitation) has been reviewed elsewhere (Birbaumer et al., 1990; Rockstroh, Elbert, Canavan, Lutzenberger, \& Birbaumer, 1989). Briefly, negative-going slow potentials such as the readiness potential or the terminal contingent negative variation appear to reflect a depolarization of apical dendrites consequent on input from thalamoreticular arousal mechanisms. These negativities signify preparation of cortical networks for information processing, and thus occur in the context of enhanced performance on tasks requiring signal detection, motor responding. and behavioral alertness (Elbert \& Rockstroh, 1987). Positive-going slow potentials, on the other hand, reflect a decrease in the polarization of the apical dendritic layer, which raises the threshold for cortical activation during a task. In the present study, slow-potential positivity was observed on

${ }^{3}$ The temporal relationships illustrated in Figure 3 with respect to $P_{Z}$ were also apparent at $C_{Z}$ and other midline locations. However, baroreceptor influences may be more prominent at sites removed from $\mathrm{C}_{\mathrm{Z}}$, because at $\mathrm{C}_{\mathrm{Z}}$ striate muscular action by the subject may contribute disproportionately to slow-potential shifts observed on blood pressure increase trials (see Birbaumer, Elbert, Canavan, \& Rockstroh, 1990). This might explain why correlations between slow potential and blood pressure differentiations tended to decrease with increasing proximity to this site. 
blood pressure increase compared to decrease trials at all midline sites, indicating a generalized disfacilitory effect. This effect might assist baroreceptormediated cardiovascular regulation by dampening sensorimotor mechanisms that contribute to total metabolic load (Skinner, 1985).

\section{Role of Learning in Hypertension}

A dampening mechanism of this type was invoked by Dworkin (1988), when he hypothesized that some cases of hypertension might be attributed to the reinforcement of pressor responses by the antinociceptive effects of cortical inhibition. Our findings support the possibility of this hypothesis, by showing that instrumentally-learned pressor responses do indeed reduce cortical arousal. It is also noteworthy that the pressor responses achieved in our study were similar in magnitude (approximately $+10 \mathrm{mmHg}$ systolic) to those observed by Larbig et al. (1985), who documented cortical disfacilitation and a reduction in pain sensitivity when human subjects were given a vasoconstrictive drug. However, it should be emphasized that the pressor responses of our study were learned through exteroceptive feedback, and not through modulation of aversive stimulation by activation of the baroreceptors. At this time it is not known whether the latter effect can intrinsically reinforce a blood pressure response. It should also be noted that antinociceptive effects induced by activation of the baroreceptors are variable between subjects and nociceptive test procedures. This variability may relate to several factors, including: 1) the tonic level of blood pressure (Elbert et al., 1988; Larbig et al., $1985), 2)$ whether a voluntary motor response is required by a nociceptive task (Randich, 1982), and/or 3) whether the conditions of the task are such that cortical modulation by the baroreceptors is itself inhibited (Spyer, 1981; Tsyrlin \& Bershadskii, 1983). These considerations call for further study of baroreceptor-mediated corticofugal effects, and of the conditions under which these effects may be discriminable and reinforcing.

Also relevant to the question of a role for learning in hypertension are the response patterns associated with instrumentally learned blood pressure responses. In the present study, pressor responses were associated with verbal reports of somatomotor, respiratory, and emotional arousal. These behavioral activities (which probably triggered the initial tachycardia on pressor trials) must have contributed to bidirectional differences in blood pressure, because in the absence of this effect there is no other basis on which to explain differential selfreport between increase and decrease $(A / B)$ trials. Some of the behaviors described by our subjects would have been expected to produce pressor responses, given the known effect of these behaviors on the circulation. Examples cited in Table 2 were a progressive tensing of leg muscles from the "big toe to the hips," which could have augmented venous filling and arterial pressures (Mancia \& Mark, 1983), and "stress/release the diaphragm" (a Valsalva-like maneuver), which could have enhanced systolic ejection in addition to stimulating cardiopulmonary baroreceptors directly (Sharpey-Schafer, 1963). Verbal reports of respiratory manipulation were corroborated by greater total respiratory power on increase compared to decrease trials $(p<$ $.01)$. However, patterns of respiratory behavior appeared to have been variable among subjects. Principal Components Analysis failed to identify consistent differences in several aspects of respiratory movement between the increase and decrease trial types.

Our findings with regard to response patterns associated with blood pressure biofeedback corroborate previous observations reported by Plumlee (1969) and Harris and Turkkan (1982). These investigators gave monkeys and baboons extensive biofeedback training in which shock avoidance was made conditional on sustained increases in blood pressure. In Plumlee's study, all monkeys were observed to contract leg extensor and arm flexor muscles by pushing down on a footrest and pulling on a head plate when pressor responses were called for. In addition, one subject was observed to engage in an apparent Valsalva maneuver on pressor trials. Harris and Turkkan (1982) described isometric "straining" against the training chair on pressor trials (p. 661), and documented an increase in plasma lactate on these trials. However, in contrast to studies by Plumlee (1969) and Harris and Turkkan (1982) in which response information was gathered through periodic visual observation by the experimenter, response information in our study was proffered by the subject in a verbal report. The presence of response information in these reports is consistent with other evidence indicating that processing of feedback behavior in attention is required for learning on feedback tasks (Hughes \& Roberts, 1985; Roberts, 1989; Roberts \& Uttl, 1990).

Although the cortical disfacilitory effect that we observed is consistent with a role for instrumental learning in idiopathic hypertension, our findings with regard to response patterns and accurate selfreport do not support the type of instrumental learning that Dworkin $(1984,1988)$ invoked to explain this disorder. Dworkin suggested that highly specific vasopressive responses are strengthened by a reinforcement mechanism that operates automatically, outside of attention. However, although 
a learning process of this type did not appear to generate the blood pressure responses we observed, learning could contribute to hypertension in other ways. For example, the behavior patterns documented here and in earlier experiments on blood pressure biofeedback (Harris \& Turkkan, 1982; Plumlee, 1969) appear to resemble bracing or straining responses of the type that are elicited when organisms are exposed to noxious stimulation. It is possible that subjects become aware of the antinociceptive effects of these behaviors, and perform the response when confronted with painful or noxious events. Repetition of bracing behavior over time might induce hypertension through luminal injury and atherosclerotic processes. Alternatively, bracing responses might be sensitized by repeated encounters with stress, or this behavior might be transferred to cues predictive of stress by Pavlovian conditioning (Rau et al., 1988). One advantage of the latter mechanisms is that behavioral adaptations achieved through them need not be processed at the level of conscious attention (Roberts, 1989). The cortical-disfacilitory effects documented in the present study are consistent with any of these accounts of how vasopressive response patterns are learned and contribute to hypertension.

In an earlier experiment that was procedurally similar to the current one, Roberts et al. (1989) gave subjects feedback for increases and decreases in slow potentials rather than for variations in blood pressure. Verbal reports of somatomotor activation were found when negative shifts in slow potentials had to be produced, whereas in the present experiment using blood pressure biofeedback the reverse relation was documented. Because the feedback procedures were isomorphic in these two experiments, this disparity can reside only in the particular patterns of behavior that subjects adopted to solve the feedback tasks. This result is noteworthy, because the presence of such patterning (including cognitive and emotional action) complicates the assessment of response specificity in biofeedback. For example, Miller and Brucker (1979) reported that quadriplegic patients given biofeedback for pressor responses exhibited smaller pressor responses when instructed to tense their muscles (paralyzed and nonparalyzed) than when instructed to increase blood pressure. This finding was taken to suggest that the pressor responses had been learned directly. However, it is not obvious that an instruction to tense one's muscles would elicit the particular pattern of integrated behavior that is optimal for increasing blood pressure. Identification of that pattern may have been assisted by Miller and Brucker's procedure, in which blood pressure feedback was provided when patients were instructed to produce pressor responses but not when patients were instructed to tense their muscles.

\section{REFERENCES}

Abboud, F.M., \& Thames, M.D. (1983). Interaction of cardiovascular reflexes in circulatory control. In J.T. Shepherd \& F.M. Abboud (Eds.), Handbook of physiology: Section 2. The cardiovascular system (pp. 675753). Bethesda, MD: American Physiological Society.

Birbaumer, N., Elbert, T., Canavan, A., \& Rockstroh, B. (1990). Slow cortical potentials of the cerebral cortex and behavior. Physiological Reviews, 70, 1-41.

Bonvallet, M., Dell, P., \& Hiebel, G. (1953). Sinus carotidien et active electrique cerebrale. C.R. Society of Biology, 147, 1166-1169.

Dworkin, B.R. (1984). Operant mechanisms in physiological regulation. In T. Elbert, B. Rockstroh, W. Lutzenberger, \& N. Birbaumer (Eds.), Self-regulation of the brain and behavior (pp. 296-309). New York: SpringerVerlag.

Dworkin, B.R. (1988). Hypertension as a learned response: The baroreceptor reinforcement hypothesis. In T. Elbert, W. Langosch, A. Steptoe, \& D. Vaitl (Eds.), Behavioral medicine in cardiovascular disorders (pp. 17-47). Chichester, UK: Wiley \& Sons.

Dworkin, B.R., Filewich, R.J., Miller, N.E., Craigmyle, N., \& Pickering, T.G. (1979). Baroreceptor activation reduces reactivity to noxious stimulation: Implications for hypertension. Science, 205, 1299-1301.

Elbert, T., Lutzenberger, W., Rockstroh, B., \& Birbaumer, N. (1985). Removal of ocular artefacts from the EEG-
A biophysical approach to the EOG. Electroencephalography \& Clinical Neurophysiology, 60, 455-463.

Elbert, T., \& Rockstroh, B. (1980). Some remarks on the development of a standardized time constant. Psychophysiology, 17, 504-505.

Elbert, T., \& Rockstroh, (1987). Threshold regulation-A key to understanding of the combined dynamics of EEG and event-related potentials. Journal of Psychophysiology, 1, 317-333.

Elbert, T., Rockstroh, B., Lutzenberger, W., Kessler, M., Pietrowsky, R., \& Birbaumer, N. (1988). Baroreceptor stimulation alters pain sensation depending on tonic blood pressure. Psychophysiology, 25, 25-29.

Garsik, J.T., Low, W.C., \& Whitehorn, D. (1983). Differences in transmission through the dorsal column nuclei in spontaneously hypertensive and Wistar Kyoto rats. Brain Research, 271, 188-192.

Harris, A.H., Gilliam, W.J., Findley, J.D., \& Brady, J.V. (1973). Instrumental conditioning of large-magnitude daily, 12-hour blood pressure elevations in the baboon. Science, 182, 175-177.

Harris, A.H., \& Turkkan, J.S. (1982). Plasma lactate levels during baseline and blood pressure conditioning in the baboon. Physiology and Behavior, 29, 657-663.

Hughes, D.E., \& Roberts, L.E. (1985). Evidence of a role for response plans and self-monitoring in biofeedback. Psychophysiology, 22, 427-439. 
Koch, E.B. (1937). Die irradiation der pressoreceptorischen Kreislaufreflexe. In A. Schweitzer (Ed.), Die irradiation autonomer Reflexe (pp. 225-227). Basel: Karger.

Larbig, W., Elbert, T., Rockstroh, B., Lutzenberger, W., \& Birbaumer, N. (1985). Elevated blood pressure and reduction of pain sensitivity. In J.F. Orlebeke, G. Mulder, \& L. van Doornen (Eds.), Psychophysiology of cardiovascular control (pp. 113-122). New York: Plenum.

Mancia, G., \& Mark, A.L. (1983). Arterial baroreflexes in humans. In J.T. Shepherd \& F.M. Abboud (Eds.), The handbook of physiology: The cardiovascular system (pp. 755-793). Baltimore, MD: Williams \& Wilkins.

Miller, N.E., \& Brucker, B.S. (1979). A learned visceral response apparently independent of skeletal ones in patients paralyzed by spinal lesions. In H.D. Kimmel \& N. Birbaumer (Eds.), Biofeedback and self-regulation (pp. 287-304). Hillsdale, NJ: Erlbaum.

Peñáz, J. (1973). Photoelectric measurement of blood pressure, volume, and flow in the finger. In R. Albert, W. Vogt, \& W. Helbig (Eds.), Digest of the 10th international conference on medical and biological engineering, Dresden, DDG.

Plumlec, L.A. (1968). Operant conditioning of blood pressure increases and decreases in the monkey. Psychophysiology, 4, 507-508.

Plumlee, L.A. (1969). Operant conditioning of increases in blood pressure. Psychophysiology, 6, 283-290.

Randich, A. (1982). Sinoartic baroreceptor reflex arc modulation of nociception in spontaneously hypertensive and normotensive rats. Physiological Psychology, 10. 267-272.

Randich, A., \& Hartunian, C. (1983). Activation of the sinaortic baroreceptor reflex arc induces analgesia: Interactions between cardiovascular and endogenous pain inhibition systems. Physiological Psychology. 11, 214-220.

Rau, H., Elbert, T., Lutzenberger, W., Eves, F., Rockstroh, B., Larbig, W., \& Birbaumer, N. (1988). Pavlovian conditioning of peripheral and central components of the baroreceptor reflex. Journal of Psychophysiology. 2, 119-127.
Roberts, L.E. (1989, November). A general associative process for Pavlovian and Thorndikian Conditioning. Paper presented at the annual meetings of the Psychonomic Society, Atlanta, GA.

Roberts, L.E., Birbaumer, N., Rockstroh, B., Lutzenberger, W., \& Elbert, T., (1989). Self-report during feedback regulation of slow cortical potentials. Psychophysiology, 26, 392-403.

Roberts, L.E., \& Uttl, B. (1990). Feedback learning under dual-task and incidental training conditions [Abstract]. Psychophysiology, 27(Suppl.), S59.

Roberts, L.E., Williams, R.J., Marlin, R.G., Farrell, T., \& Imiolo, D. (1984). Awareness of the response after feedback training for changes in heart rate and sudomotor laterality. Journal of Experimental Psychology: General, 113, 225-255.

Rockstroh, B., Elbert, T., Canavan, A.G.M., Lutzenberger, W., \& Birbaumer, N. (1989). Slow cortical potentials and behavior ( 2 nd ed.). Munich: Urban \& Schwarzenberg.

Sharpey-Schafer, E.P. (1963). Effect of respiratory acts on the circulation. In W.F. Hamilton (Ed.), Handbook of physiology: Section 2. Circulation (Vol. III, pp. 18751886). Washington, DC: American Physiological Society.

Skinner, J. (1985). Regulation of cardiac vulnerability by the cerebral defense system. Journal of the American College of Cardiology. 5/6(Suppl.), 88-94.

Spyer, K.M. (1981). Neural organization and control of the baroreceptor reflex. Reviews of Physiology. Biochemistry, and Pharmacology. 88, 32-124.

Tsyrlin, V.A., \& Bershadskii, B.G. (1983). Mechanisms of hypertensive responses to emotional stress. Soviet Neurology and Psychiatry. 16, 64-73.

Victor, R., Weipert, D., \& Shapiro, D. (1984). Voluntary control of systolic blood pressure during postural change. Psychophysiology. 21, 673-681.

Wessling, K.H., Settels, J.J., \& de Wit, B. (1986). The measurement of continuous finger arterial pressure noninvasively in stationary subjects. In T.H. Schmidt, T.M. Dembroski, \& G. Blumchen (Eds.), Biological and psychological factors in cardiovascular disease (pp. 355-375). Heidelberg: Springer-Verlag. 